\title{
IMECE2006-14735
}

\section{DEVELOPMENT OF A STANDARD FOR NEW PASSENGER CAR WHEEL DESIGNS}

\author{
Brandon Talamini \\ U.S. DOT \\ Volpe Center \\ Cambridge, MA \\ talamini@volpe.dot.gov
}

\author{
Benjamin Perlman \\ Dept. of Mechanical Engineering \\ Tufts University \\ Medford, MA 02155 USA \\ bperlman@tufts.edu
}

\author{
Jeff Gordon \\ U.S. DOT \\ Volpe Center \\ Cambridge, MA \\ gordonj@volpe.dot.gov
}

\section{ABSTRACT}

The American Public Transportation Association (APTA) is seeking to develop specifications to ensure that wheels used in transit and commuter applications perform safely under the service conditions to which they are exposed. To this end, a design standard has been conceived to ensure that new wheel designs proposed for such applications are not susceptible to fatigue cracking in the wheel plate and hub.

Historically, the Association of American Railroads (AAR) Standard S-660 has been applied in the industry for the purposes of qualifying wheel designs for use in passenger applications. The standard stipulates particular loads to apply in a simple finite element analysis of the new wheel design. The basis for approval is an empirical comparison (by an independent third party) of the results with those in a database of previous analysis results of other qualified wheels.

The proposed "S-660 equivalent" design standard is envisioned to be self-qualifying, in that results of the analysis will directly determine whether the wheel design will perform safely in service; a review or approval body will not be required. The new standard is needed to overcome limitations embodied in the current wheel qualification process, namely, the assumption of purely elastic material behavior, the omission of residual stresses due to manufacturing, and the use of comparative approval criteria.

The Union Internationale des Chemins de Fer (UIC) introduced a wheel design requirement based on finite element analysis, the results of which are subjected to a fatigue criterion in order to achieve acceptance of the wheel design. As in the current S-660 methodology, a set of thermal and mechanical loads are prescribed. This methodology is essentially self-qualifying as the results of the analysis (obtained following a prescribed procedure) determine whether the wheel design will perform safely in service.

The proposed design standard is envisioned to be a combination of the current S-660 analysis requirements and the fatigue calculation-based approach of the UIC. The task force developing the standard is still resolving the specific details of the thermal and mechanical loading requirements.

This paper explores the underlying methodology behind the developing standard. A finite element calculation forms the basis of the qualification procedure. Initial (asmanufactured) residual stresses present in a new wheel are determined. Mechanical and thermal loading representative of passenger operations are applied. The analysis yields three characteristic stress distributions: as-manufactured, mechanical, and thermal. The Sines criterion, with temperature-dependent material fatigue properties obtained from testing, is applied to infer whether the candidate wheel design is fatigue-prone. Results are presented for a wheel design currently in transit/commuter service. The APTA committee is currently investigating the thermal and mechanical load levels to be prescribed in the proposed standard.

\section{INTRODUCTION}

The goal of this work is to begin to formalize the procedures to be specified in a developing APTA wheel design standard. The new standard is under consideration as a replacement for the current AAR Standard S-660 [1]. The S660 standard specifies the conduct of specific finite element analyses using prescribed loading conditions. The loading specified in the S-660 standard, however, is more relevant to freight than passenger applications. Specifically, the thermal 
loading requirement represents a drag-braking scenario, more common in freight than passenger operations. Further, the current S-660 has several shortcomings:

1. The analysis assumes fully elastic conditions.

2. No allowance is made for the presence of beneficial compressive residual stress that is imparted during manufacture.

3. The basis for approval is an empirical comparison. Approval for use of the wheel in service is granted following favorable comparison (by an independent third party) of results of this analysis with those in a database of previous analysis results for other wheels.

In order to develop a revised wheel qualification procedure, a methodology must be developed to overcome these limitations. This paper seeks to address these issues. First, a modeling technique is proposed to quantify the stresses in new wheel designs. The as-manufactured residual stresses are developed from an elastic-plastic finite element analysis. Next, service stresses resulting from the specific operating conditions to which the subject wheel is to be exposed are estimated. These are dependent on the characteristics of the passenger vehicle with which the wheel is to be used and the performance demand expected in service. Finally, the influence of the overall stress state (initial, asmanufactured stresses plus live service stresses) on the likelihood of the new wheel design to experience premature fatigue failure is determined. To accomplish this, the revised wheel design standard is envisioned to be self-qualifying in that independent review of the results will not be required. Instead, application of a fatigue criterion is being considered, whereby the adequacy of the wheel design can be demonstrated using the results of the prescribed analysis. These procedures are under investigation as means to overcome the three limitations identified above.

\section{SINES CRITERION FOR FATIGUE}

The Sines criterion [2] has been selected for use as the indicator of the likelihood that a particular wheel design is fatigue-prone. The Sines criterion is attractive because it deals with combined stress states and can account for the effect of static (residual) stresses. The Sines criterion is described by the following equation:

$\frac{1}{3} \sqrt{\left(P_{1}-P_{2}\right)^{2}+\left(P_{2}-P_{3}\right)^{2}+\left(P_{1}-P_{3}\right)^{2}}-A-\alpha\left(S_{x}+S_{y}+S_{z}\right) \leq 0$

where

$P_{1}, P_{2}, P_{3}=$ amplitudes of the alternating principle stresses,

$S_{x}, S_{y}, S_{z}=$ normal components of mean stress,

$A=$ material constant proportional to reversed fatigue strength, and

$\alpha=$ material constant, which gives variation of the permissible range of stress with static stress.
Application of the Sines criterion in the context of the subject wheel analysis is a multi-step process requiring numerical estimates of three independent stress distributions. The alternating principle stresses $\left(P_{i}\right)$ are those due to repeated wheel-rail contact cycles and thermal stresses from brake applications. The $P_{i}$ are obtained from elastic finite element analysis solutions with prescribed contact or braking loads. The mean stresses $\left(S_{x, y, z}\right)$ are the as-manufactured residual stresses generated by an elastic-plastic finite element simulation of the wheel heat treatment process. When the criterion is not satisfied (i.e., the effective alternating stresses dominate the static stresses), fatigue failure can be expected before the desired lifetime.

The desired lifetime is expressed by the constants $A$ and $\alpha$, which describe the fatigue properties of the material. $A$ is a measure of the fatigue strength for fully reversed stress cycles; $\alpha$ accounts for the inverse effect of mean stress on fatigue life. The constants can be determined from any two sets of fatigue tests in which the mean stresses are appreciably different. Convenient candidates are the reversed axial test and the zerotension fluctuating stress test. Constants $A$ and $\alpha$ have been determined experimentally for AAR Class A and B wheel steel material at ambient and elevated temperature [3, 4]. The results of these experiments are shown in Figure 1.

The APTA committee developing the revised standard has determined that the Sines criterion is to be applied in regions of the wheel cross-section that are remote from the locations where thermal loading from friction braking occurs. This is due primarily to the fact that localized heating of the wheel rim during friction braking can often result in significant thermal stresses, which may be manifested as violations of the fatigue criterion. The potential for wheel tread thermal cracking has been addressed in a separate APTA Recommended Practice described in [5].

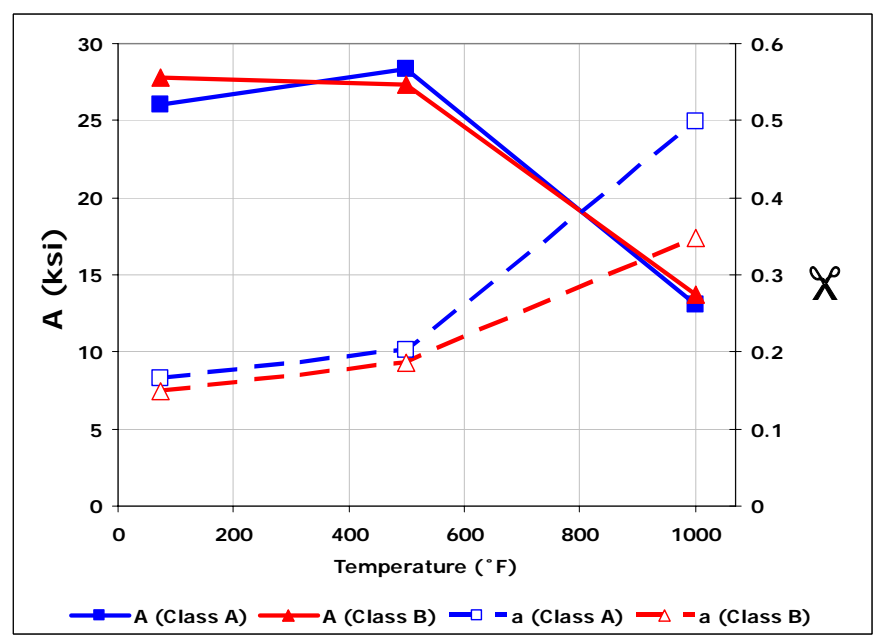

Figure 1. Sines parameters $A$ and $\alpha$ for AAR Class $A$ and $B$ wheel steel as function of temperature. 


\section{MODELING CONSIDERATIONS}

Since this study seeks to develop a standardized procedure for the conduct of the analysis, the potential complexity and labor intensity of the methodology has been given consideration. Previous work has focused on application of axisymmetric models to estimate residual stresses due to heat treatment and thermal loading from braking [6]. Contact loads were not considered. In order to properly account for wheel/rail contact effects a threedimensional model is necessary. Furthermore, in the spirit of developing a useful alternative wheel design standard, an integrated modeling approach is desired.

As noted above, application of the Sines criterion requires knowledge of the distribution of stresses from three different sources: manufacturing, contact, and brake shoe heating. Since it is necessary that these stresses be known at the same locations within the cross-section, it is logical to consider conducting the analysis using a single three-dimensional model. However, three-dimensional simulation of the wheel manufacturing process is extremely time-consuming and unnecessary since the process is axisymmetric. Since the criterion treats these stress distributions separately, it is not critical that they be obtained from the same model, only that they be available at the same cross-sectional locations.

For the manufacturing simulation a two-dimensional (axisymmetric) model is constructed from the $45^{\circ}$ plane of a quarter-symmetric three-dimensional model. In this way, the nodes in each model have common coordinates, and the stress distributions obtained from each can be easily incorporated into the Sines criterion.

A 36 in $(91.5 \mathrm{~cm})$ reverse dish passenger car wheel is chosen for the prototype analysis. A three-dimensional model is constructed for use with the ABAQUS [7] finite element analysis program, as shown in Figure 2. This $90^{\circ}$ quartersymmetric model will be used to conduct the contact simulations and comprises 48,618 nodes and 43,308 elements. A two-dimensional model mesh is extracted at the $45^{\circ}$ midplane of the three-dimensional model. The two-dimensional model, shown in Figure 3, comprises 1,261 nodes and 1,159 elements, and is used to conduct the wheel heat treatment simulation. For convenience, the two-dimensional model is also used for the braking simulation. (Brake shoe heating is not a two-dimensional phenomenon. In the future, thermal stresses from braking will be investigated using the threedimensional model). Element density is increased in the wheel rim in order to better resolve the stress gradients that are expected to exist in the vicinity of the wheel tread.

\section{MANUFACTURING PROCESS SIMULATION}

The first step in the analysis sequence is calculation of the as-manufactured residual stress distribution. Pertinent parameters relevant to the process, such as wheel initial temperature, location and duration of quenchant application, and annealing temperature and duration, are obtained from the wheel supplier.
The quenching and annealing portions of the wheel manufacturing process were simulated following the general procedure described in [8]. The wheel is initially at $1600^{\circ} \mathrm{F}$ $\left(871^{\circ} \mathrm{C}\right)$. The tread region only is then quenched for 2.5 minutes with a water spray. Next, the wheel is annealed at $810^{\circ} \mathrm{F}\left(432^{\circ} \mathrm{C}\right)$ for four hours, after which it cools to room temperature. Appropriate boundary conditions are prescribed to account for convective and radiative heat losses. Temperature-dependent thermomechanical material properties are employed.

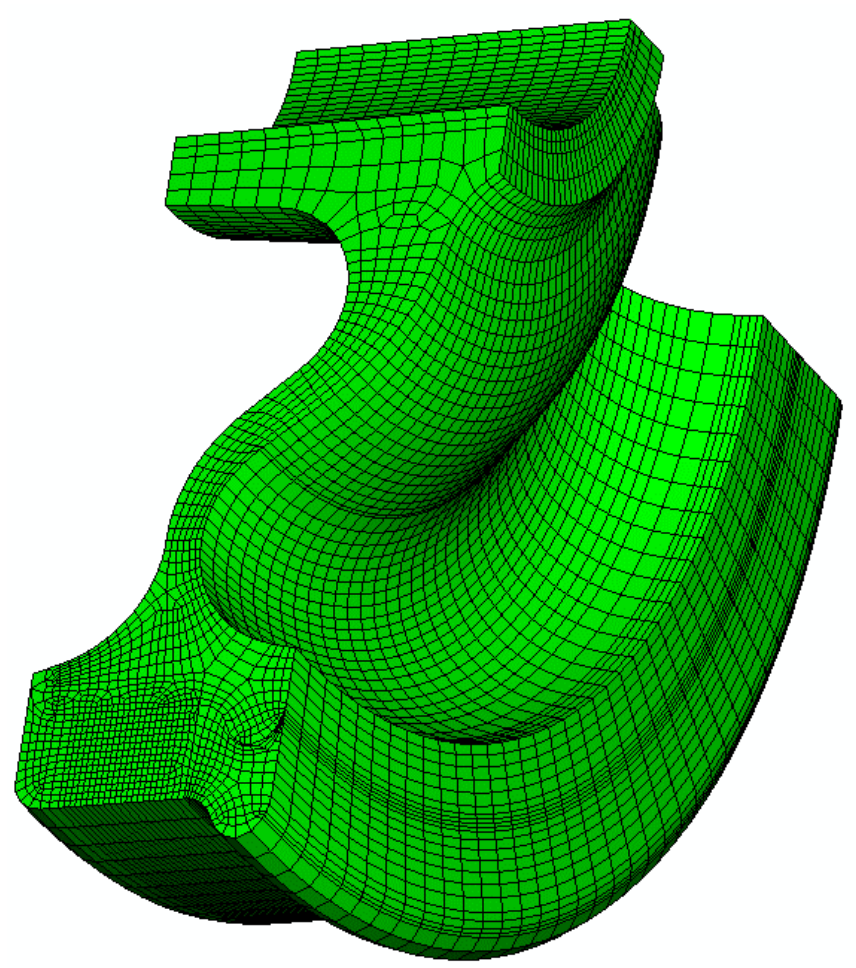

Figure 2. Three-dimensional wheel model.

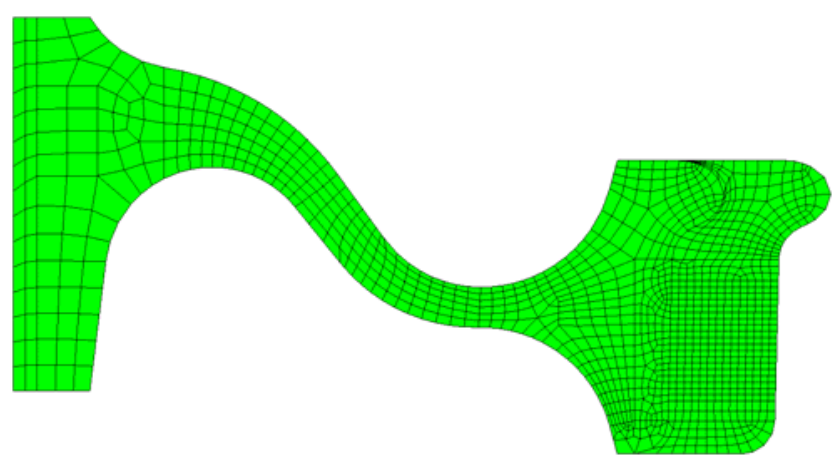

Figure 3. Two-dimensional wheel model representing cross-section on $45^{\circ}$ plane. 
The most important result of the simulated heat treatment is shown in Figure 4. Contours are presented in ksi $(1 \mathrm{ksi}=$ $6.895 \mathrm{MPa})$ throughout the paper. Residual circumferential (hoop) compression extends from the wheel tread surface to a depth of approximately 1.25 in $(3.2 \mathrm{~cm})$ into the rim. The presence of this hoop compression increases resistance to the formation and growth of cracks at the wheel tread. A more useful way to portray these results is shown in Figure 5, which is a plot of the residual hoop stress along a line extending from the tread surface into the wheel rim. For comparison, the figure also includes results obtained from a three-dimensional heat treatment simulation (using the model depicted in Figure 2). Agreement between the two models is very good; however, the three-dimensional heat treatment model requires approximately 48 hours to execute while the two-dimensional equivalent requires 9 minutes. From the point of view of the eventual design standard, the axisymmetric simplification for the simulated manufacturing portion of the analysis represents a significant improvement in efficiency.
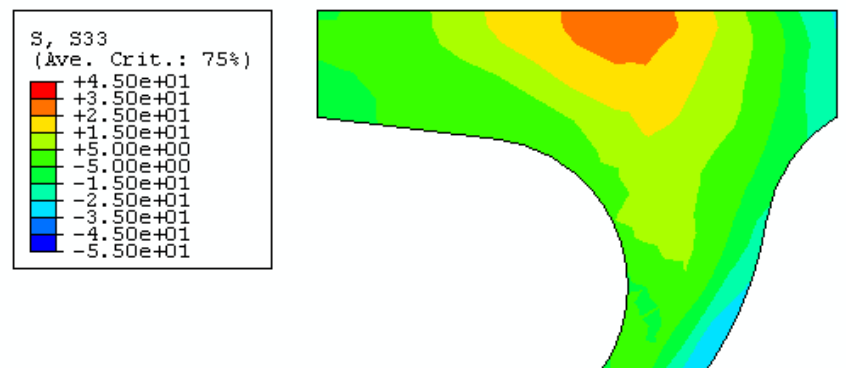

Figure 4. Contour plot of residual hoop stress in new wheel following simulated manufacturing.

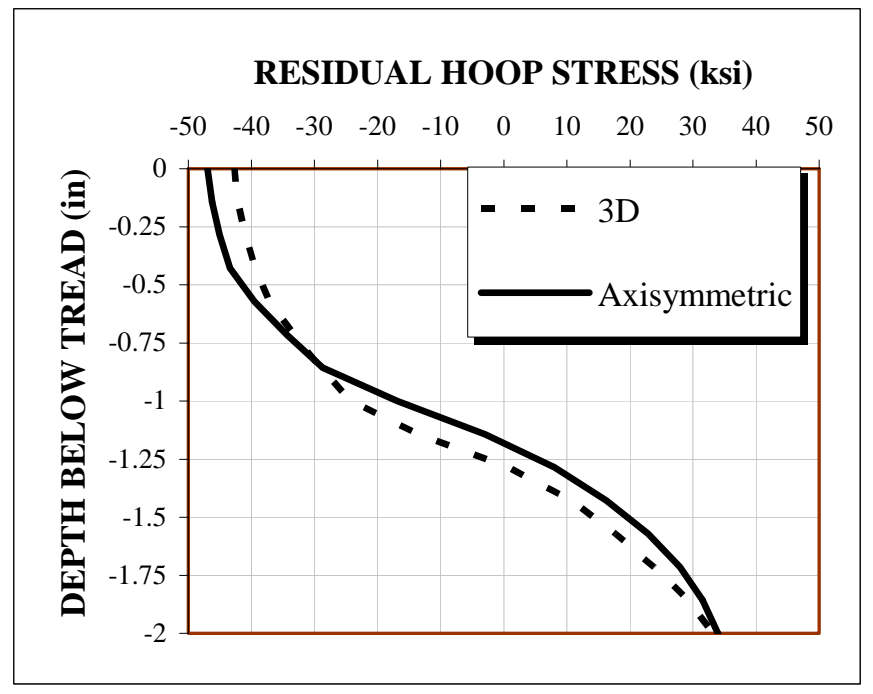

Figure 5. Comparison of two-dimensional and threedimensional heat treatment models (line plot through rim at center of tread).

\section{CONTACT LOADING}

In this prototype study, contact loading is considered in a simplified manner in order to maintain some consistency with the legacy S-660 standard. More realistic wheel-rail contact characterization is considered in a companion paper in this conference [9]. AAR Standard S-660 specifies locations on the wheel tread at which vertical and lateral loads are applied simulating wheel-rail contact. Since the Sines criterion is to be evaluated at regions away from the wheel tread, the mechanical loads are applied as discrete point loads. S-660 requires application of vertical and lateral loads equal to $2 P$ and $P$, respectively, where $P$ is the nominal static wheel load. This loading condition is deemed by the APTA standard development committee to be unnecessarily large, especially when considering passenger wheel loading. For the purposes of this preliminary study, vertical loading is assumed to equal $P$ and lateral loading has a value of $0.6 P$. Load magnitude will be varied in future investigations in order to identify an appropriate specification for the eventual design standard. The subject vehicle weight is $180,000 \mathrm{lb}(800 \mathrm{kN})$ yielding a static wheel load $P$ of $22,500 \mathrm{lb}(100 \mathrm{kN})$. The loads (L1, V1) are applied at positions corresponding to those specified in the S-660 standard: both loads are applied to a point 1.5 in (3.8 $\mathrm{cm}$ ) to the field side from the outer gage side of the flange. Figure 6 shows the resulting von Mises stresses due to the combined vertical and lateral load. 

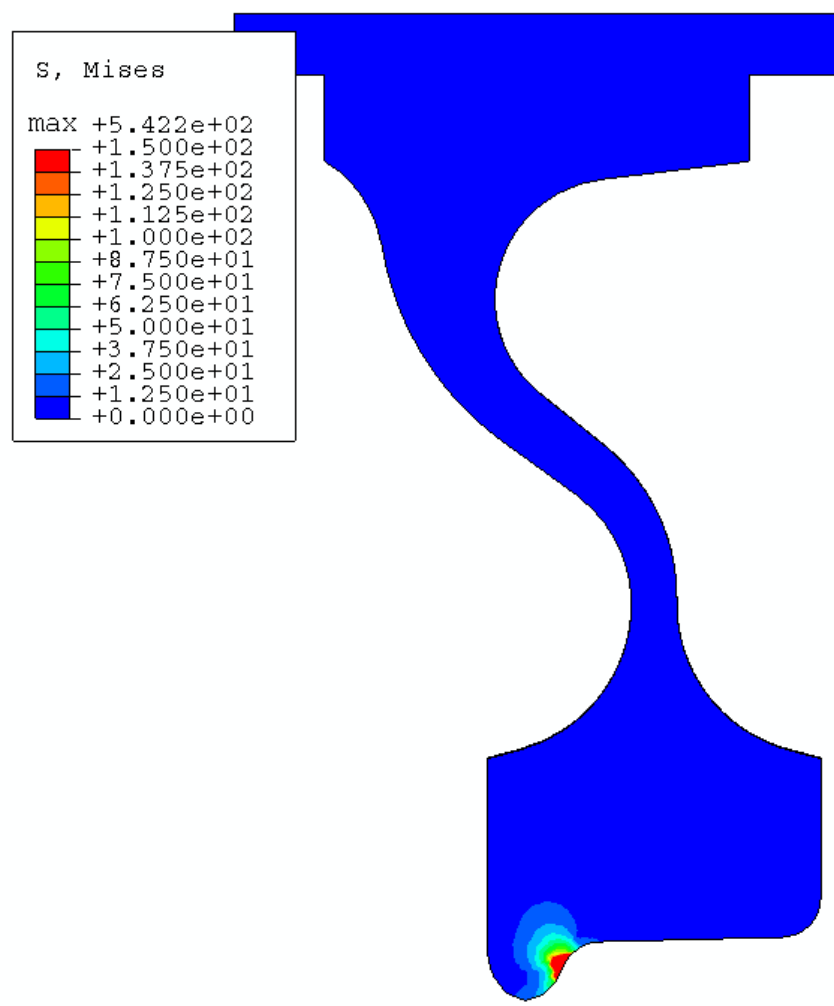

Figure 6. Von Mises stress in wheel cross-section due to contact loading.

The results in Figure 6 indicate very localized contact effects for the applied load magnitude. In the immediate vicinity of the point of application of the load, stresses are extremely large. This is due primarily to the fact that the loads are applied at a single node and not distributed over a contact patch as illustrated in [9]. More importantly, there are no regions of stress concentration anywhere else in the crosssection. This implies that the wheel plate and hub fillet regions are not likely to be susceptible to fatigue due to contact loading alone.

\section{THERMAL LOADING}

Wheel thermal loading is implemented as a timedependent heat flux applied to the wheel tread surface over an area approximating that of a brake shoe. The magnitude of the heat flux is determined based on the assumed vehicle weight, the initial speed (100 mph; $160 \mathrm{~km} / \mathrm{hr})$, and the deceleration rate $(2 \mathrm{mph} / \mathrm{sec} ; 3.2 \mathrm{~km} / \mathrm{hr} / \mathrm{sec})$. Under these conditions, the theoretical vehicle stop time is 50 seconds. These parameters yield a maximum heat flux of 1.0935 BTU/in ${ }^{2}$-s $\left(1.7880 \mathrm{MW} / \mathrm{m}^{2}\right)$, which decreases linearly to zero during the simulated stop, forming a triangular heat flux distribution. The vehicle is assumed to make 20 service stops (triangular flux loading repeated 20 times) with 11 minutes between each stop. In order to reduce computation time, the same axisymmetric model used for the heat treatment simulation is used for the thermal analysis. The flux is distributed over a 2.5 in $(6.35 \mathrm{~cm})$ band centered on the wheel tread.
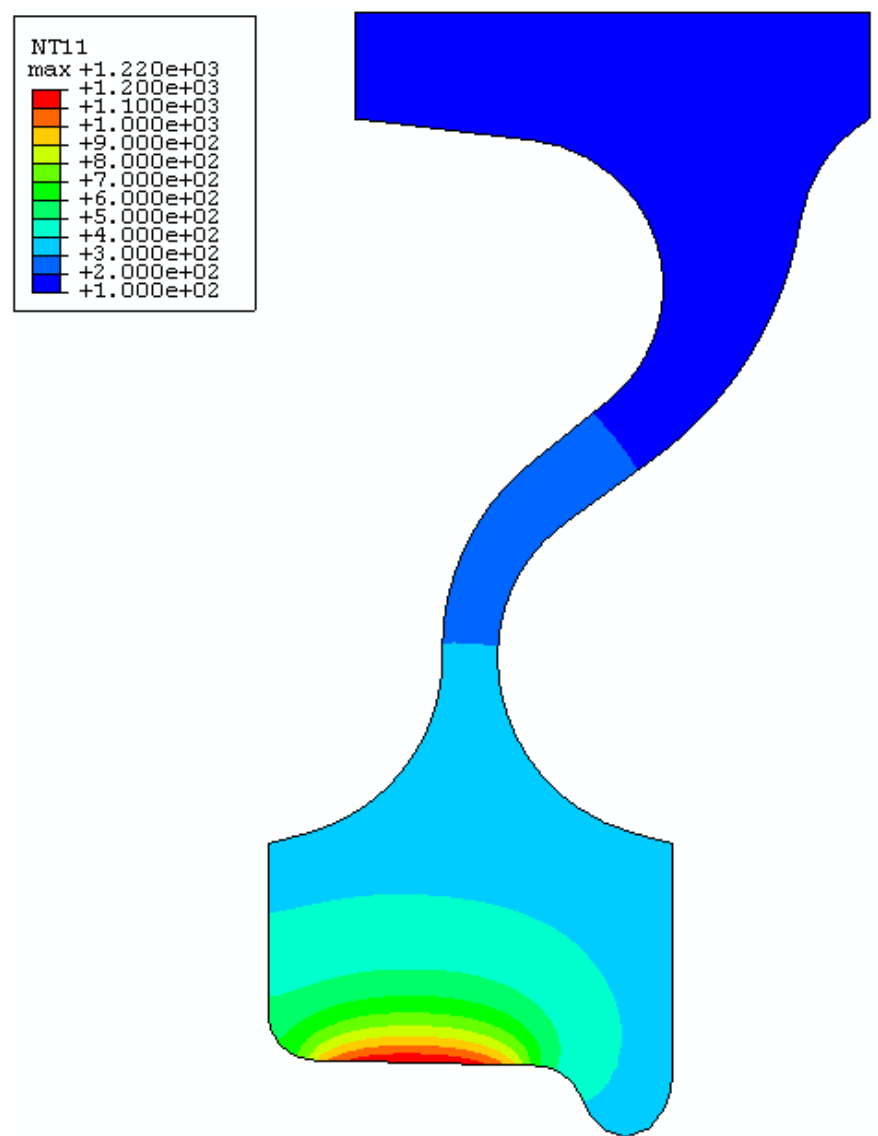

Figure 7. Temperature distribution during $20^{\text {th }}$ simulated service stop.

Elastic thermal stresses are extracted from the model when the surface temperature reaches its extreme value during the last stop of the simulated revenue run. Figure 8 shows the thermal hoop stress distribution.

Figure 7 suggests that wheel surface temperatures can exceed $1200^{\circ} \mathrm{F}\left(650^{\circ} \mathrm{C}\right)$. A very shallow region near the wheel tread is subjected to such high temperature. An increase in temperature is observed deeper into the rim, which is manifested by the hoop stress gradient shown in Figure 8. 


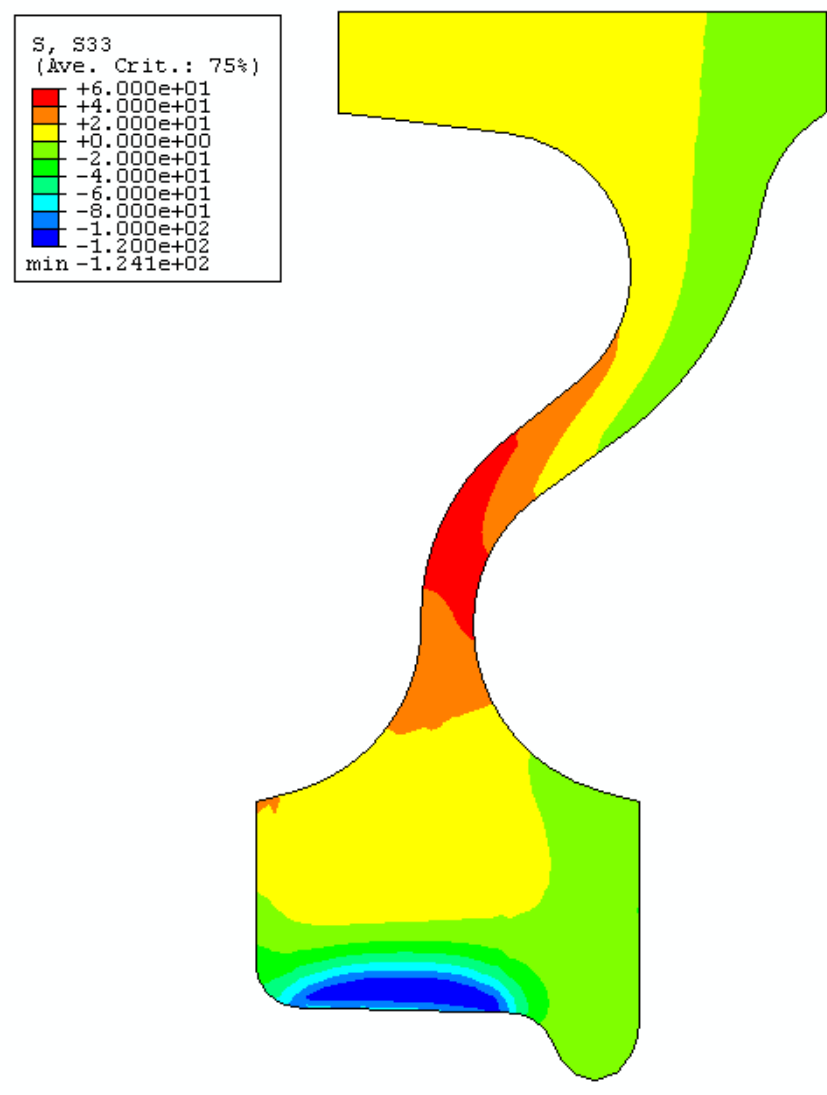

Figure 8. Maximum elastic hoop stress contours during $20^{\text {th }}$ simulated stop.

\section{RESULTS OF APPLICATION OF SINES CRITERION}

Please note that the loading conditions assumed for this prototype calculation are ad-hoc and have been chosen for illustrative purposes only. Specifically, the thermal duty cycle applied to this wheel is intentionally extremely severe. These results are not to be construed as suggesting that the subject wheel design is fatigue-prone. The authors wish to make clear that this paper presents a proposed methodology and is not intended as a critique of a particular wheel design or manufacturer.

The prototype procedure yields three stress distributions: the as-manufactured residual stresses, stresses from wheel/rail contact, and thermal stresses from friction braking. Stresses are collected for each node on the $45^{\circ}$ plane for the threedimensional contact calculation and from each corresponding node in the axisymmetric model for the heat treatment and braking simulations.

For each node, the stresses for each of these states are stored in ASCII files. These are then read by a MathCad [10] procedure that performs the following functions:
1. Each of the three stress distributions is read into an array indexed by node number.

2. $S_{x, y, z}$ are obtained from the as-manufactured stress distribution for each node.

3. The thermal and mechanical stresses are combined and the eigenvalues are determined to generate the principle alternating stresses $\left(P_{i}\right)$.

4. Based on the nodal temperature, the appropriate values for $\mathrm{A}$ and $\alpha$ are interpolated using the data from Figure 1.

5. For each node, the Sines equation is evaluated.

6. The data generated in step 6 are plotted as a contour plot. Positive contours indicate locations where fatigue is likely within the desired lifetime.

Figure 9 shows a typical result (as described in step 6). Regions depicted in other than medium blue are locations at which the Sines criterion is violated, thus identifying locations at which premature fatigue failure is likely. Figure 9 suggests that under the loading conditions assumed, fatigue cracking in the wheel rim/plate fillet region may occur during the design life of the wheel. The location where the wheel/rail contact load is applied is also highlighted as fatigue-prone in Figure 9. This is an artifact of having applied the loads at a single point. Actual wheel/rail contact would result in a distributed load over a contact patch.

As noted above, the loading conditions assumed for this demonstration are intentionally severe. The proposed methodology is intended to be applied to new wheel designs only, not wheel designs that have been approved for service under the S-660 procedure.

In order to illustrate the effect on the results, reduced thermal input is illustrated in Figure 10. In this example, the thermal load is reduced to that corresponding to simulated 80 mph (128 km/hr) operation. The maximum heat flux is now $0.8748 \mathrm{BTU} / \mathrm{in}^{2}-\mathrm{s}\left(1.4304 \mathrm{MW} / \mathrm{m}^{2}\right)$ and the stop time is reduced to 40 seconds. The plate no longer contains any predicted fatigue-prone locations.

A means to apply the method to a wheel design that is not likely to perform well in service can be devised by excluding the beneficial residual stresses imparted to the wheel during the manufacturing process. Figure 11 presents the results of the Sines criterion evaluation applied to $80 \mathrm{mph}(128 \mathrm{~km} / \mathrm{hr})$ service when the manufacturing stresses are ignored. In this case the number of locations at which premature fatigue failure can be expected is increased. Indeed, the tread region in the vicinity of the brake shoe is now included. This result illustrates the versatility of the Sines criterion in this application. 


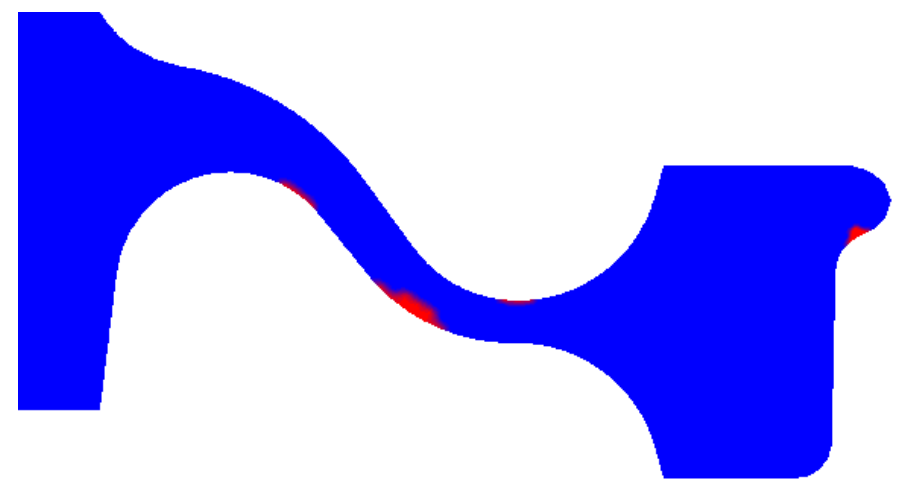

Figure 9. Typical contour plot of results of evaluation of Sines criterion at an operating speed of $100 \mathrm{mph}$ (160 $\mathrm{km} / \mathrm{hr}$ ). Regions depicted in other than medium blue indicate locations at which Sines criterion is not satisfied.

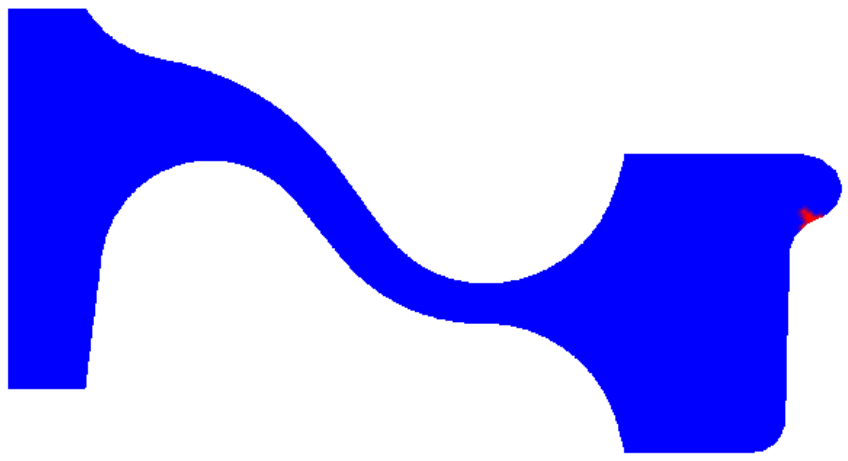

Figure 10. Contour plot of results of evaluation of Sines criterion at reduced speed operation at $80 \mathrm{mph}$ (128 $\mathrm{km} / \mathrm{hr}$ ). Regions depicted in other than medium blue indicate locations at which Sines criterion is not satisfied.

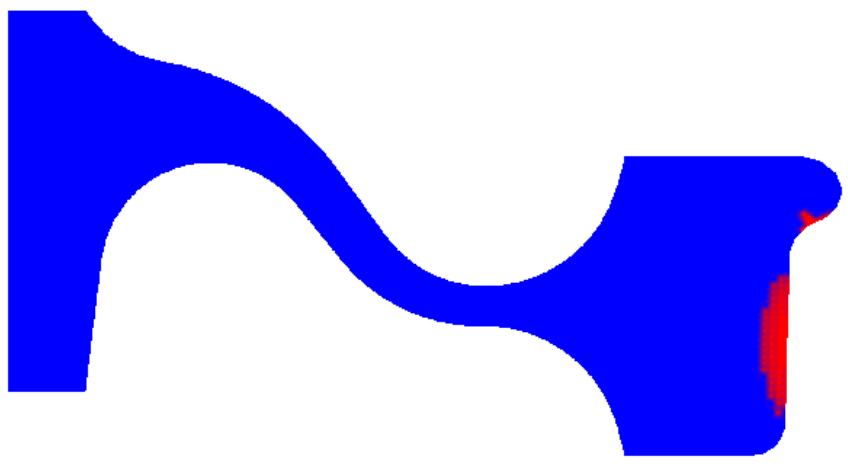

Figure 11. Contour plot of results of evaluation of Sines criterion when initial, as-manufactured residual stresses are ignored, assuming reduced speed operation at $80 \mathrm{mph}$ $(128 \mathrm{~km} / \mathrm{hr})$. Regions depicted in other than medium blue indicate locations at which Sines criterion is not satisfied.

\section{CONCLUSIONS}

This paper has outlined an analysis procedure, which is intended to form the basis for an eventual wheel design standard to replace the currently applied AAR S-660. The proposed technique has been demonstrated on a wheel design that is common in transit/commuter service. This prototype calculation demonstrates the ability to use the analysis to make reasonable estimates. This work has demonstrated the ability to migrate from the S-660 freight-focused approach to account for the differences that arise in passenger service. In this case, the braking cycles are intermittent rather than continual (as in drag braking) and the wheel/rail contact is less important (but needs to be taken into consideration).

To fully implement this prototype calculation as a design standard, some additional work must be done to ensure that the procedure is reliable in the hands of a competent analyst. Precise model requirements (including element size, mesh density, etc.) have yet to be considered. The Sines fatigue parameters (A and $\alpha$ ) are currently only available for AAR Class A and B wheel steel. Testing to determine these constants for AAR Class $\mathrm{C}$ material is under consideration. Current assumptions related to the service braking duty cycle may warrant revision.

\section{ACKNOWLEDGMENTS}

The work reported in this paper was carried out under the Rail Equipment Safety program sponsored by the Office of Research and Development, Federal Railroad Administration, U.S. Department of Transportation, under the direction of Ms. Claire L. Orth, Chief, Equipment and Operating Practices Research Division. Ms. Monique Stewart is the Project Manager for the research related to railroad wheel safety.

\section{REFERENCES}

[1] Procedure for the Analytic Evaluation of Locomotive and Freight Car Wheel Designs, Association of American Railroads Standard S-660, Adopted 1981, Revised 2004.

[2] Sines, G., "Behavior of Metals Under Complex Static and Alternating Stresses,” Metal Fatigue, G. Sines and J. L. Waisman, Eds., McGraw-Hill, NY, pp.145-169, 1959.

[3] McMaster, F., Robledo, G.P. and Gordon, J.E., "Fatigue Performance of AAR Class A Railroad Wheel Steel at Ambient and Elevated Temperatures," Proc. International Mechanical Engineering Congress and Exhibition, ASME RTD, 2005.

[4] McMaster, F. and McKeighan, P., "Fatigue Behavior of Railcar Wheel Steel at Ambient and Elevated Temperature," Southwest Research Institute, San Antonio, TX, August 2003. DOT/FRA/ORD-03/19. http://www.volpe.dot.gov/sdd/docs /fail/fatgbehavior.pdf

[5] Gordon, J. and Stone, D.H., "Development of a Passenger Wheel Standard," Proc. International Mechanical Engineering Congress and Exhibition, ASME RTD 2005.

[6] Gordon, J. and Perlman, A.B., "Effects of Wear and Service Conditions on Residual Stresses in Commuter 
Car Wheels," Proc. $14^{\text {th }}$ International Wheelset Congress, Orlando, FL, November 17-21, 2004.

[7] Abaqus/Standard User's Manual, Volume I and II (Version 6.5-1). Hibbitt, Karlsson and Sorenson, Inc., Warwick, RI, 2004.

[8] Gordon, J., "Estimation of Residual Stresses in Railroad Car Wheels Resulting from Manufacture and Service Loading," MS Thesis, Tufts University, Medford, MA, February 1998.

[9] Liu, S., Perlman, A.B., and Gordon, J.E., "Residual Stresses in Passenger Car Wheels," Proc. International Mechanical Engineering Congress and Exhibition, ASME RTD, 2006.

[10] MathCad, Version 11.0. Mathsoft Engineering and Education, Inc., 2002. 